\title{
Trattamento con etanercept dei soggetti con artrite reumatoide in Italia: considerazioni economiche
}

Lorenzo G. Mantovani*

\begin{abstract}
Rheumatoid arthritis is a chronic inflammatory disease affecting the joints and leading to work disability in half of the patients; its prevalence in Italy is estimated to be around $0,5 \%$. The understanding of the pathophysiology of the disease has lead to the development of biotechnologically-derived drugs, among which anti-TNF- $\alpha$ agents. At the present moment, two of these drugs are available in Italy: etanercept and infliximab.

The aim of the present work was to furnish a pharmaco-economical comparison among the two anti- TNF- $\alpha$ agents in Italy. The efficacy of antirheumatic therapies is usually measured in terms of percentage of responders, defined as those patients whose symptoms improve of at least 20, 50 or 70\% (ACR20, ACR50, ACR70). A review of the published literature revealed that, according to these accepted parameters, etanercept results superior when infliximab is administered at standard doses, while it is at least as effective as infliximab when the latter is at the highest doses.

Although drug acquisition costs are comparable for the two drugs at the suggested regimens, economical evaluations performed in several countries indicate that total treatment costs are lower with etanercept as compared to infliximab, mainly because of differences in the route of administration (subcutaneous vs. slow IV infusion), need for patient monitoring and co-administration of methotrexate with infliximab and days at work gained.

Thus, from this preliminary analysis it appears that etanercept dominates infliximab, i.e. it provides better results at a lower cost, but this result needs to be tested and confirmed by prospective economical evaluations.
\end{abstract}

Farmeconomia e percorsi terapeutici 2003; 4 (1): 33-38

\section{INTRODUZIONE}

L'artrite reumatoide (AR) è una malattia infiammatoria cronica caratterizzata da dolore, disabilità funzionale e distruzione delle strutture articolari.

A 10 anni dalla diagnosi quasi il 50\% dei pazienti risulta inabile al lavoro [1]. La mortalità dei soggetti affetti da AR è significativamente più elevata di quella della popolazione generale [2, 3]. In Italia si stima che la prevalenza dell' AR sia lo $0.5 \%$ (circa 300.000 malati).

Le recenti acquisizioni sul processo reumatoide nelle sue diverse componenti di flogosi, proliferazione sinoviale e distruzione articolare hanno permesso di mettere a punto farmaci, ottenuti mediante biotecnologie, selettivamente mirati ai meccanismi patogenetici della malattia. Svariati farmaci biologici potenzialmente attivi nell'AR sono attualmente in fase di studio. Tra questi, gli agenti in grado di bloccare il TNF- $\alpha$ (etanercept e infliximab) sono quelli maggiormente sperimentati e autorizzati per uso clinico in Italia.

Risulta pertanto interessante valutare il profilo farmacoeconomico degli anti-TNF- $\alpha$, con particolare riferimento ad etanercept.

\section{LETTERATURAECONOMICA DELL'USODIETANERCEPT}

Esistono diversi lavori pubblicati circa gli aspetti economici della terapia con anti-TNF- $\alpha$.

Uno studio farmacoeconomico olandese [4] ha confrontato i costi annuali totali attribuibili a etanercept e infliximab in pazienti adulti affetti da artrite reumatoide. I risultati del lavoro (espressi nel lavoro originale in fiorini olandesi) riportano un minore consumo di risorse economiche in seguito alla somministrazione di etanercept in monoterapia rispetto a 
infliximab (in combinazione con methotrexate). L'evidenza proposta da Nujiten e colleghi mostra come a fronte di costi della terapia farmacologica sovrapponibili (14.200-14.300 euro circa), la terapia con infliximab risulta più costosa a motivo del maggior consumo di altre risorse sanitarie (visite, diagnostica, terapie concomitanti, day-hospital etc.) necessarie per la somminstrazione endovenosa di infliximab, la quale richiede l'accesso ospedaliero del paziente. I costi aggiuntivi annui della terapia con infliximab rispetto a etanercept sono quantificabili in circa 5.500 euro, a carico del terzo pagante. Tali stime risultano conservative, in quanto Nujiten e colleghi non considerano l'escalation nel dosaggio di infliximab.

Il National Institute for Clinical Excellence (NICE) britannico, in "Guidance on the use of Etanercept and Infliximab for the treatment of Rheumatoid Arthritis" [5], raccomandando l'uso dei nuovi farmaci in Europa per la terapia dell' artrite reumatoide nei bambini e negli adulti, pubblica i risultati di una valutazione economica sull'impatto dei farmaci anti-TNF (infliximab, etanercept) nel trattamento dell' artrite reumatoide.

L'analisi di costo-utilità incrementale, effettuata mediante un modello preliminare che considerava i costi derivanti dal farmaco, ma non i risparmi in termini di ospedalizzazione, di trasporto, di produttività e di morte, mostrava un costo pari a 63,974£ per QALY (QualityAdjusted life-year) guadagnato per etanercept e pari a 99,373£ per infliximab.

Un'analisi costo-utilità, elaborata con un modello più ampio proposto da Wyeth, ha fornito per etanercept un valore di costo-efficacia incrementale di 16,330£ per QALY. Un'analisi simile, con un modello proposto da ScheringPlough, ha fornito perinfliximab un valore costo-efficacia incrementale di 23,936£ per QALY.

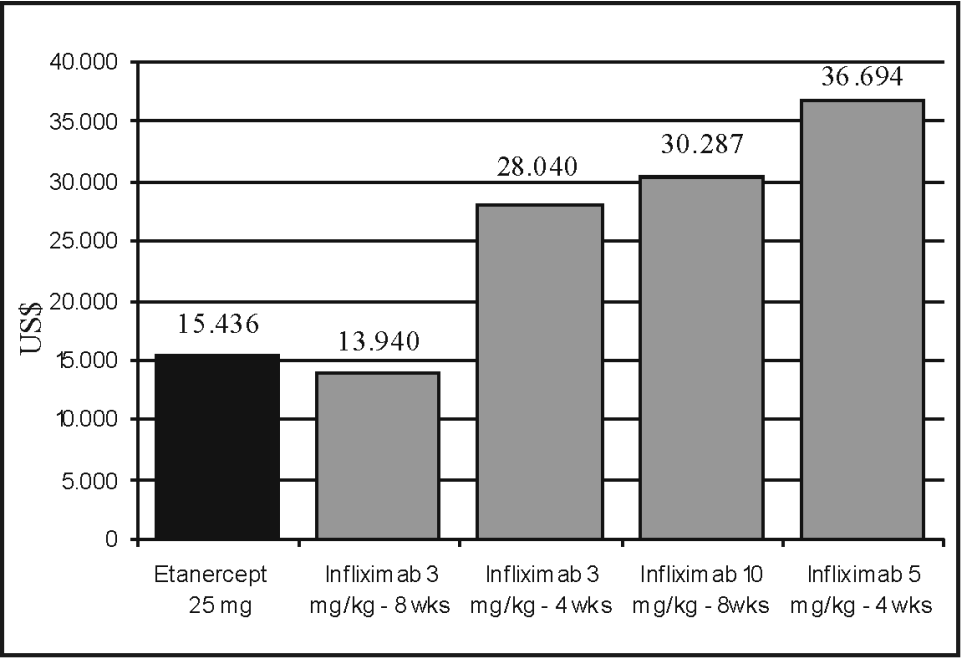

Figura 1

Costo annuo della terapia con anti-TNF-a negli Stati Uniti (US\$)
Queste discrepanze hanno portato il NICE a rivedere le proprie analisi. È stata pertanto effettuata a cura del NICE una rivalutazione dell'analisi costo-utilità fornita dalle Case Produttrici, considerando valori più conservativi e restrittivi per le variabili. I risultati hanno mostrato un valore di costo-utilità incrementale di 27,000£ per QALY per etanercept e di $35,000 £$ per QALY per infliximab. Questi ultimi valori sono quelli utilizzati dal NICE nel prendere la propria decisione (positiva) riguardo alla terapia con anti-TNF- $\alpha$.

Diverse valutazioni economiche dell'utilizzo di anti-TNF- $\alpha$ sono state presentate durante il Congresso dell'EULAR nel 2002. I punti che da esse emergono sono i seguenti. Innanzitutto la terapia con anti-TNF- $\alpha$ è in assoluto in grado di generare un minor consumo di risorse sanitarie [6], particolarmente nei soggetti sottoposti a terapia con etanercept vs infliximab, a motivo della necessità della somministrazione di quest'ultimo in regime di day-hospital [7]. In secondo luogo, la terapia con anti-TNF- $\alpha$ genera un miglioramento marcato dello status lavorativo dei soggetti che la ricevono in termini sia di giornate lavorate, sia di partecipazione alla forza lavoro [6, 8-9]. Tale miglioramento è comunque più marcato per etanercept, vista la possibilità di effettuare la terapia a domicilio, evitando di perdere giornate lavorative a motivo della somministrazione, come invece accade per infliximab. Infine la terapia con anti-TNF- $\alpha$ mostra valori di costo efficacia e di costo utilità che rientrano nei parametri giudicati accettabili dalla comunità scientifica, particolarmente quando vengono presi in considerazioni i costi indiretti (status lavorativo). In tale ambito, etanercept mostra un miglior profilo di costo-efficacia e costoutilità sia in assoluto sia in comparazione ad infliximab [10-13].

Un lavoro pubblicato sul numero del febbraio 2002 di Arthritis \& Rheumatism, "Guidelines for the management of Rheumatoid Arthritis: 2002 Update" [14], riporta il confronto tra i costi annuali della terapia effettuata con infliximab ed etanercept, con l'esame di diversi dosaggi di infliximab. Questi dati non tengono conto del costo aggiuntivo del metotressato. I risultati, in dollari, sono visualizzati nella figura 1 .

\section{EFFICACIADELL'USODI ETANERCEPT}

I risultati dei trials clinici fino ad ora riportati hanno dimostrato l'efficacia del trattamento con anti-TNF-a. Esiste, di fatto, una tendenza ad una efficacia inferiore del dosaggio più basso di infliximab ( $3 \mathrm{mg} / \mathrm{kg}$ ogni 8 settimane) sia rispetto all'alto dosaggio dello stesso far- 
maco $(10 \mathrm{mg} / \mathrm{kg})$ sia, indirettamente, rispetto ad etanercept [15-19]. Le tabelle 1 e 2 mostrano l'efficacia di etanercept e infliximab nel generare una risposta secondo i criteri ACR 20, 50, 70 a 6 mesi e un anno, in diverse tipologie di pazienti con AR (precoce, attiva) con e senza (per etanercept) la terapia concomitante con metotressato.

\section{COSTODELLATERAPIACONANTI-TNF- $\propto$}

Infliximab si somministra per infusione lenta (circa tre ore). Questo comporta l'ospedalizzazione del paziente (anche in day hospital) con relativi costi diretti di screening (radiografia, tine-test, ECG) e somministrazione. Ciò si traduce, nell'ottica dell'ospedale, in un impegno aggiuntivo del personale medico/paramedico specializzato, del farmacista che prepara il farmaco prima della somministrazione, del reumatologo, dell'anestesista. Nella prospettiva del Servizio Sanitario Nazionale, la necessità della somministrazione in regime ospedaliero si traduce nel costo di un DRG, o di DH o di regime ordinario.

Al contrario, etanercept si somministra per via sottocutanea anche a domicilio del paziente, in modo più semplice, economico e, prevedilmente, meglio accettato dal paziente.
Il costo totale della terapia dipende, oltre che dal costo diretto del farmaco, anche da altri costi, quali l'eventuale associazione con altri farmaci, il monitoraggio della tollerabilità, la somministrazione.

Etanercept è indicato ad una dose fissa di $25 \mathrm{mg}$ due volte alla settimana (Riassunto delle caratteristiche del prodotto). Il costo di una fiala è 127,56 euro, mentre il costo annuale del farmaco risulta essere di 13.260 euro circa.

Etanercept può essere usato in monoterapia e non si hanno perciò i costi di una terapia aggiuntiva. Inoltre non sono previsti controlli strumentali (radiografia, ECG) o analisi di laboratorio "extra routine" per valutarne la tollerabilità. Infine la somministrazione di etanercept, per via sottocutanea, può essere effettuata a domicilio del paziente. Non richiede, perciò, il ricovero ospedaliero, con conseguente impegno di personale specializzato.

La tabella 3 riassume il costo della terapia con etanercept, facendo riferimento al protocollo dello studio ANTARES.

Infliximab è stato utilizzato negli studi sull' artrite reumatoide ad una dose variabile dai 3 ai $10 \mathrm{mg}$ per $\mathrm{kg}$ di peso (tempo 0, 2, 6 settimane, quindi ogni 4-8 settimane).

Alcuni studi esteri hanno registrato la necessità di un'escalation nel dosaggio di infliximab nei pazienti non responders.

\begin{tabular}{|c|c|c|c|c|c|c|c|}
\hline \multirow[t]{2}{*}{ Criterio } & \multirow{2}{*}{$\begin{array}{c}\text { Etanercept } \\
25 \mathrm{mg} \\
2 \text { volte per } \\
\text { settimana }\end{array}$} & \multirow[t]{2}{*}{ Fonte } & \multicolumn{4}{|c|}{ Infliximab } & \multirow[t]{2}{*}{ Fonte } \\
\hline & & & $\begin{array}{c}3 \mathrm{mg} / \mathrm{kg} \\
\text { ogni } 8 \\
\text { settimane }\end{array}$ & $\begin{array}{c}3 \mathrm{mg} / \mathrm{kg} \\
\text { ogni } 4 \\
\text { settimane }\end{array}$ & $\begin{array}{c}10 \mathrm{mg} / \mathrm{kg} \\
\text { ogni } 8 \\
\text { settimane }\end{array}$ & $\begin{array}{c}10 \mathrm{mg} / \mathrm{kg} \\
\text { ogni } 4 \\
\text { settimane }\end{array}$ & \\
\hline $\begin{array}{l}\text { ACR20 } \\
\text { (1 anno) }\end{array}$ & $62 \%$ & [15] & $42 \%$ & $48 \%$ & $59 \%$ & $59 \%$ & [16] \\
\hline $\begin{array}{l}\text { ACR50 } \\
\text { (1 anno) }\end{array}$ & $45 \%$ & {$[15]$} & $21 \%$ & $34 \%$ & $39 \%$ & $38 \%$ & [16] \\
\hline $\begin{array}{l}\text { ACR70 } \\
\text { (1 anno) }\end{array}$ & $25 \%$ & [15] & $10 \%$ & $17 \%$ & $25 \%$ & $19 \%$ & [16] \\
\hline
\end{tabular}

\section{Tabella 1}

Frequenzadi responders ad un anno secondo i criteri ACR20, ACR50, ACR70

\begin{tabular}{|c|c|c|c|c|c|c|c|}
\hline \multirow[t]{2}{*}{ Criterio } & \multirow{2}{*}{$\begin{array}{c}\text { Etanercept } \\
25 \mathrm{mg} 2 \\
\text { volte per } \\
\text { settimana }\end{array}$} & \multirow[t]{2}{*}{ Fonte } & \multicolumn{4}{|c|}{ Infliximab } & \multirow[t]{2}{*}{ Fonte } \\
\hline & & & $\begin{array}{c}3 \mathrm{mg} / \mathrm{kg} \\
\text { ogni } 8 \\
\text { settimane }\end{array}$ & $\begin{array}{c}3 \mathrm{mg} / \mathrm{kg} \\
\text { ogni } 4 \\
\text { settimane }\end{array}$ & $\begin{array}{c}10 \mathrm{mg} / \mathrm{kg} \\
\text { ogni } 8 \\
\text { settimane }\end{array}$ & $\begin{array}{c}10 \mathrm{mg} / \mathrm{kg} \\
\text { ogni } 4 \\
\text { settimane }\end{array}$ & \\
\hline $\begin{array}{l}\text { ACR20 } \\
(6 \text { mesi) }\end{array}$ & $\begin{array}{l}59 \% \\
71 \%\end{array}$ & $\begin{array}{l}{[17]} \\
{[18]}\end{array}$ & $50 \%$ & $53 \%$ & $52 \%$ & $58 \%$ & [19] \\
\hline $\begin{array}{l}\text { ACR50 } \\
\text { (6 mesi) }\end{array}$ & $\begin{array}{l}40 \% \\
39 \%\end{array}$ & $\begin{array}{l}{[17]} \\
{[18]}\end{array}$ & $27 \%$ & $29 \%$ & $31 \%$ & $26 \%$ & [19] \\
\hline $\begin{array}{l}\text { ACR70 } \\
(6 \text { mesi) }\end{array}$ & $\begin{array}{l}15 \% \\
15 \%\end{array}$ & $\begin{array}{l}{[17]} \\
{[18]}\end{array}$ & $8 \%$ & $11 \%$ & $18 \%$ & $11 \%$ & [19] \\
\hline
\end{tabular}

\section{Tabella 2}

Frequenza di responders a sei mesi secondo i criteri ACR20, ACR50, ACR70 
Durez e colleghi hanno aumentato, a partire dalla ventiduesima settimana e in $1 / 4$ dei pazienti, la dose in misura pari ad una fiala per paziente per somministrazione, mantenendo la frequenza di somministrazione [20]. Nello stesso studio, dei pazienti ancora in trattamento originario un ulteriore $24,2 \%$ e $9,2 \%$ di pazienti alla quarantaseiesima e sessantaduesima hanno aumentato la dose.

Dati simili sono stati riportati dallo studio STURE: le dosi iniziali di $3 \mathrm{mg} / \mathrm{kg}$ sono state aumentate a $5-7 \mathrm{mg} / \mathrm{kg}$ in circa il $45 \%$ dei pazienti complessivamente [21]. Va segnalato che le dosi accresciute vengono mantenute anche durante i periodi successivi, aumentando, prevedibilmente, i costi negli anni successivi al primo.

L'esperienza derivante dalla pratica clinica in Italia, e riportata in alcuni abstract del $38^{\circ}$ Congresso SIR 2001 [22-24], conferma la necessità dell' aumento del dosaggio pro chilo e/ o della riduzione della frequenza delle somministrazioni e della riduzione dell'effetto clinico nel lungo periodo di terapia con infliximab. Nello specifico, Fantini e colleghi hanno somministrato infliximab ogni 6 settimane dopo il periodo di induzione, vale a dire a partire dalla dalla 14esima settimana.

Sulla base di questi dati è stata effettuata una valutazione dei costi annui della terapia con infliximab. Il protocollo di Fantini e colleghi corrisponde ad un numero di 10 somministrazioni per paziente. In questo protocollo non è stata riportata alcuna escalation nel dosaggio di Infliximab. Per calcolare il numero di fiale utilizzate si suppone che il $75 \%$ dei pa- zienti abbia un peso corporeo inferiore ai $70 \mathrm{~kg}$ richiedendo 2 fiale per somministrazione e che il restante $25 \%$ richieda 3 fiale a somministrazione.

A tali costi sono stati aggunti i costi dei DRG relativi ai day hospital necessari per la somminstrazione di infliximab ed i costi della terapia con metotressato (formulazione orale, ipotesi conservativa).

È stata inoltre effettuata un' analisi per verificare quali siano/sarebbero i costi della somministrazione di infliximab come riportato nello studio STURE [21], vale a dire ipotizzando un dosaggio iniziale di $3 \mathrm{mg} / \mathrm{kg}$ con escalation terapeutica fino a $5-7 \mathrm{mg} / \mathrm{kg}$, mantenendo la frequenza ogni 8 settimane durante la fase di mantenimento. Anche in questo caso, al fine di calcolare il numero di fiale utilizzate si suppone che il $75 \%$ dei pazienti abbia un peso corporeo inferiore ai $70 \mathrm{~kg}$ richiedendo 2 fiale per somministrazione e che il restante $25 \%$ richieda 3 fiale a somministrazione.

La tabella 3 riporta i risultati relativi ad una coorte ipotetica di 100 pazienti per gruppo.

\section{CONCLUSIONI}

In virtù delle analisi presentate, non è possibile calcolare un rapporto di costo-efficacia incrementale di etanercept vs infliximab, in quanto la terapia con etanercept risulta contemporaneamente sia meno costosa sia più efficace. Essa appare, in altri termini, dominante.

La terapia con etanercept appare ugualmente o più efficace rispetto a quella con infliximab
Tabella 3

Costi (in euro) del primo anno di terapia con anti-TNF- $\alpha$ in 100 pazienti italiani $\S$

\begin{tabular}{lrrr}
\hline & Etanercept & Infliximab [22]* & Infliximab STURE\# \\
\hline Numero Fiale & 10.400 & 2.200 & 2.204 \\
Costo per fiala & 127,5 & 570 & 570 \\
Costo parziale & 1.326 .000 & 1.254 .000 & 1.256 .280 \\
Day Hospital & 200 & 1.000 & 800 \\
Costo Day Hospital@ & 241 & 241 & 241 \\
Costo parziale & 44.200 & 241.000 & 192.800 \\
Metotressato & ---- & 100 & 100 \\
Costo terapia & ---- & 54 & 54 \\
Costo parziale & ---- & 5.400 & 5.400 \\
Costo totale & 1.374 .000 & 1.466 .660 & 1.454 .480 \\
\hline
\end{tabular}

$\S$ Si suppone che il $75 \%$ dei pazienti abbia un peso corporeo inferiore ai $70 \mathrm{Kg}$ richiedendo 2 fiale per somministrazione e che il restante $25 \%$ richieda 3 fiale a somministrazione

* Si suppone che la somministrazione avvenga al dosaggio di $3 \mathrm{mg} / \mathrm{Kg}$ durante tutto il periodo e che a partire dal termine del periodo di induzione (45-esimo giorno) la terapia di mantenimento venga somministrata ogni 45 giorni.

\# Si suppone che Infliximab venga somministrato ogni 8 settimane durante tutto il periodo di mantenimento, aumentando il dosaggio nei non responders a $5-7 \mathrm{mg} / \mathrm{Kg}(6 \mathrm{mg} / \mathrm{Kg}$ in media).

@ Quantificato utilizzando la tariffa nazionale per il day hospital reumatologico. 
quando questa è prescritta ai dosaggi minimi, mentre risulta simile ai dosaggi più elevati di infliximab.

Il costo della terapia con etanercept è superiore al costo di quella con infliximab solo quando questo è somministrato ai dosaggi minimi (3 $\mathrm{mg} / \mathrm{kg}$, ogni 8 settimane in mantenimento), caso che appare irrealistico nella pratica per:

1) minor incidenza di responders ACR20 a tali dosaggi/frequenza di somministrazione;

2) difficoltà nella divisione delle unità posologiche di infliximab.
Nelle condizioni reali di utilizzo ( $3 \mathrm{mg} / \mathrm{kg}$ ogni 6 settimane, escalation del dosaggio a $5-7 \mathrm{mg}$ / $\mathrm{kg}$ nei non responders), il costo della terapia con infliximab è superiore a quello di etanercept (dose fissa di $25 \mathrm{mg} 2$ volte la settimana).

In tali condizioni di utilizzo, la terapia con etanercept appare dominante rispetto a quella con infliximab, in quanto meno costosa e maggiormente o ugualmente efficace.

Questi risultati preliminari andranno comunque verificati e confermati da studi economici prospettici.

\section{BIBLIOGRAFIA}

1. Wolfe F et al. The long term outcomes of rheumatoid arthritis. Arthritis Rheum 1998; 41: 1072-82

2. Wolfe F et al. The mortality of rheumatoid arthritis. Arthritis Rheum 1994; 37: 481-9

3. Pincus T. The underestimated long term medical and economic consequences of RA. Drugs 1995; 50 (suppl 1): 1-74.

4. Nujiten MJC, Engelfriet P, Duijn $\mathrm{K}$ et al. A cost-cost study comparing etanercept with infliximab in rheumatoid arthritis. Pharmacoeconomics 2001; 19:1051-64.

5. National Institute for Clinical Excellence (NICE). Guidance on the use of Etanercept and Infliximab for the treatment of Rheumatoid Arthritis, March 2002. Disponibile all'indirizzo web: www.nice.org.uk

6. Yelin. EH, Katz PP, Lubeck DP et al. Impact of Etanercept (enbrel) on health care utilization and employment among persons with early rheumatoid arthritis. Disponibile all'indirizzo web: http://www.eular.org/eular2002/ abstract.cfm

7. Geborek P, Eberhardt K, Saxne T. Etanercept and infliximab treatment reduces hospital care in arthritis patients. Disponibile all'indirizzo web: http://www.eular.org/eular2002/abstract.cfm.

8. Geborek P, Eberhardt K, Larsson B, et al. Etanercept and infliximab treatment improves working capacity in arthritis patients. Disponibile via URL all'indirizzo web: http://www.eular.org/eular2002/abstract.cfm.

9. Van Vollenhoven R F, Harju A, Bratt J. Treatment with infliximab or etanercept results in significant increases in work force participation: data from the Stockholm TNF-alpha antagonist registry (sture). Disponibile all'indirizzo web: http://www.eular.org/eular2002/abstract.cfm.

10. Bansback N, Bansback, Brennan A, Conway P, Reynolds A. The cost-effectiveness of 3rd line etanercept versus other dmards in adults with rheumatoid arthritis in the UK. Disponibile all'indirizzo web: http://www.eular.org/ eular2002/abstract.cfm.

11. Bansback N, Bansback, Brennan A, Conway P, Reynolds A. Impact of direct and indirect costs on the costeffectiveness of etanercept in rheumatoid arthritis in the UK. Disponibile all'indirizzo web: http://www.eular.org/ eular2002/abstract.cfm.

12. Malone DC, OrtmeierBG. Cost efficacy of etanercept versus infliximab plus methotrexate in rheumatoid arthritis based on radiographic data. Disponibile all'indirizzo web: http://www.eular.org/eular2002/abstract.cfm.

13. Malone DC, Ortmeier BG. Cost efficacy of etanercept versus infliximab plus methotrexate in the treatment of dmardresistant rheumatoid arthritis. Disponibile all'indirizzo web: http://www.eular.org/eular2002/abstract.cfm.

14. American College of Rheumatology. Guidelines for the management of Rheumatoid Arthritis, 2002 Update. Arthr Rheum 2002, 46: 328-346.

15. Bathon JM, Martin RW, Fleischmann RM et al. A comparison of Etanercept and Methotrexate in patients with early rheumatoid arthritis. NEJM 2000; 343: 1586-93.

16. Lipsky PE, vdHejide DMFM, St Clair EW et al. Infliximab and methotrexate in the treatment of rhematoid arthritis. NEJM 2000; 343: 1594-602.

17. Moreland LW, Schiff MH, Baumgartner SW et al. Etanercept therapy in RA: a randomized controlled study. Ann Intern Med 1999; 130:478-86. 
Trattamento con etanercept dei soggetti con artrite reumatoide in Italia: considerazioni economiche

18. Weinblatt ME, Kremer JM, Bankhurst AD et al. A trial of etanercept, a recombinant tumor necrosis factor receptor: $F c$ fusion protein, in patients with $R A$ receiving methotrexate. NEJM 1999; 340:253-59.

19. Maini R, St Clair EW, Breedveld F et al. Infliximab vs placebo in RA patients receiving concomitant methotrexate: a randomized phase III trial. Lancet 1999; 354: 1932-1939.

20. Durez P, Devogelaer JP, Van den Bosch F et al. Infliximab and methotrexate in the treatment of rheumatoid arthritis: clinical results in a belgian observational cohort.EULAR 2002. Disponibile all'indirizzo web: http://www.eular.org/ eular2002/abstract.cfm.

21. Van Vollenhoven RF, Gullstrom E, Brannemark S, Klareskog L. Dose escalation of Infliximab in clinical practice: data from the Stockholm TNF-alpha Registry (STURE), EULAR Congress 2002. Disponibile all'indirizzo web: http://www.eular.org/eular2002/abstract.cfm.

22. Fantini F, Tosi S, Sinigallia L, et al. Risultati ad un anno del trattamento con infliximab in pazienti affetti da artrite reumatoide refrattaria. Reumatismo 2001, 53 suppl. 4: 324.

23. Botsios C, Sfriso P, Ostuni PA. Applicazione e risultati preliminari del protocollo di studio osservazionale Antares in 44 pazienti trattati con infliximab. Reumatismo 2001, 53 suppl. 4: 324.

24. Covelli M, Scioscia C, Compagno M et al. Studio aperto sulla tollerabilità ed efficacia di un trattamento combinato con methotrexate ed infliximab in poliartriti croniche non responders: dati ad 1 anno e follow-up. Reumatismo 2001, 53 suppl. 4: 325 . 